\title{
Risques pluviométriques, source d'insécurité alimentaire et nutritionnelle au Niger
}

\author{
Tahirou HAWEY ${ }^{1,2 *}$, Lawali SITOU ${ }^{3}$, Abdou Maman MANSSOUR ${ }^{1}$, \\ Idrissa SOUMANA ${ }^{2}$, Moussa MASSAOUDOU² et Alzouma Mayaki ZOUBEIROU ${ }^{1}$ \\ ${ }^{I}$ Département Production Durable des Cultures, Faculté des Sciences Agronomiques, Université Boubacar BA \\ de Tillabéri, BP 175 Tillabéri Niger. \\ ${ }^{2}$ Département d'Economie Rurale, Sociologie et transfert des technlogies, Institut National de la Recherche \\ Agronomique du Niger (INRAN) BP 429 Niamey, Niger. \\ ${ }^{3}$ UMR : Développement durable, Sociétés et Adaptation aux Changements Climatiques, Faculté d'Agronomie \\ et des Sciences de l'environnement, Université Dan Dicko Dankoulodo de Maradi, BP: 465 Maradi, Niger. \\ *Auteur correspondant ; E-mail: haweytahirou79@gmail.com
}

\section{RESUME}

Au cours des dernières décennies, le Niger a subi des nombreux aléas climatiques qui ont pour conséquences une diminution de la production agricole et une recrudescence des crises alimentaires et nutritionnelles. L'objectif de cette étude, vise à analyser le lien entre l'insécurité alimentaire nutritionnelle et les risques pluviométriques. La méthodologie utilisée est surtout basée sur des enquêtes (entretiens en groupe et entretiens individuels) dont les populations cibles sont celles des régions de Maradi, Tahoua et Tillabéri. Les résultats relèvent qu'il existe une différence significative entre les précipitations des trois régions. Les résultats, ont aussi, montré que les deux risques pluviométriques sont : les sécheresses et les inondations. Mais, au vu de la population rurale, les sécheresses impactent beaucoup négativement sur la sécurité alimentaire et nutritionnelle car elles engendrent une diminution de la production céréalière et une augmentation des prix des céréales locaux. La sécheresse contribue fortement au déterminisme de l'insécurité alimentaire et nutritionnelle au Niger. (C) 2020 International Formulae Group. All rights reserved.

Mots clés : Risques, sécheresse, inondation, sécurité alimentaire et nutritionnelle.

\section{Rainfall risks, source of food and nutritional insecurity in Niger}

\begin{abstract}
In recent decades, Niger has suffered from numerous climatic hazards which have resulted in a decrease in agricultural production and a resurgence of food and nutrition crises. The objective of this study is to analyze the link between nutritional food insecurity and rainfall risks. The methodology used is mainly based on surveys (group interviews and individual interviews) whose target populations are those of the regions of Maradi, Tahoua and Tillabéri. The results show that there is a significant difference between the precipitation in the three regions. The results also showed that the two rainfall risks are: droughts and floods. However, in view of the rural population, droughts have a very negative impact on food and nutrition security because they cause a reduction in cereal production and an increase in local cereal prices. Drought contributes significantly to the determinism of food and nutritional insecurity in Niger.
\end{abstract}

(C) 2020 International Formulae Group. All rights reserved.

Keywords: Risks, drought, flood, food security and nutritional. 


\section{INTRODUCTION}

Pays sahélien et aride, le Niger est confronté à une pluviométrie annuelle comprise entre $100 \mathrm{~mm}$ et $800 \mathrm{~mm}$ caractérisée par une importante variabilité interannuelle et spatiale avec en conséquences de sécheresses et/ou des inondations (Véronique, 2014). Le pays compte une seule saison de pluies, ou est concentré, pour les paysans, l'essentiel des activités agricoles. Les précipitations constituent l'un des facteurs climatiques le plus important fragilisant ainsi l'équilibre environnemental (Idrissa, 2008).

La production céréalière est fortement dépendante des précipitations. Cette production souligne également que, les conditions favorables à l'installation et à la bonne croissance des cultures céréalières sont la phase d'installation et de fin de la campagne, périodes qui déterminent aussi la longueur de la saison agricole (Bacci et al., 2014). Une baisse des précipitations en ces moments critiques engendre un risque pour le complément du cycle végétatif des cultures notamment le mil et le sorgho qui sont les deux principales céréales au Niger tant du point de vue superficies emblavées que de leur contribution dans l'alimentation. Ainsi, ces deux cultures sont cultivées dans les trois régions et occupent $70 \%$ dans la consommation alimentaire (Songré et al., 2015).

Pour toutes les cultures, les pertes de production résultant d'un incident climatique peuvent être quantitatives et/ou qualitatives en relation avec les précipitations (Sall, 2015). Les températures les plus considérables sont aussi enregistrées pendant la saison pluviale, c'est-à-dire les températures moyennes des mois de juillet-aout et la température maximale du mois de Juin Bacci et al. (2014).

Pour les agro- phyto-écologistes, les insuffisances pluviométriques ont toujours favorisé les attaques des ennemis de cultures notamment les ravageurs et les chenilles provoquant des baisses des productions agricoles et des crises alimentaires. Ainsi, une production céréalière de quantité et de qualité passe nécessairement par la maitrise des maladies et ravageurs (Afrique Verte, 2013). A titre illustratif en 2004 au Niger, la production agricole céréalière a été sévèrement affectée par l'invasion acridienne et la sécheresse (PAM/FAO, 2004). On note en effet, l'attaque des insectes comme la cicadelle sur le mil et le sorgho, qui a causé d'énormes dégâts Haougui et al., (2017). Cependant, dans la Région de Tillabéri (Vieri et Maurizio, 2016) soulignent que c'est le risque d'inondation qui a affecté beaucoup plus les populations et les productions agricoles. Mais, la sécheresse est l'une des catastrophes naturelles les plus dévastatrices paralysant la production alimentaire, provoquant l'épuisement des pâturages, la désorganisation des marchés, et, à l'extrême, causant de nombreuses pertes en vies humaines et animales. Les sécheresses peuvent également conduire à un accroissement des migrations de population des zones rurales vers les zones urbaines ce qui exerce des pressions sur une production alimentaire affaiblie (Fao, 2020).

Des liens ont été aussi établis entre cette baisse de production et l'augmentation des prix des céréales à l'insécurité alimentaire. Les économistes comme (Kako, 2000) ont cherché les causes de la fluctuation des prix du côté de l'insuffisance de l'offre vivrière ou celle de la demande solvable des produits vivriers. L'insécurité alimentaire des petits producteurs est due à leurs systèmes de production qui restent tributaires des conditions climatiques et agro-écologiques (Boussard, 2010) et la volatilité des marchés agricoles. Les sécheresses et les épizooties sont les deux phénomènes exogènes préjudiciables aux agriculteurs et aux consommateurs.

L'objectif global de cette étude est d'identifier les variabilités pluviométriques source d'insécurité alimentaire et nutritionnelle récurrente au Niger. Les objectifs spécifiques consistent à : analyser la pluviométrie de façon 
détaillée, identifier les risques et analyser leurs conséquences socio-économiques.

\section{MATERIEL ET METHODES \\ Zone d'étude}

L'étude a été menée au Niger dans les régions de Maradi, Tahoua et Tillabéri. Sur le plan agro-écologique, ces trois régions appartiennent à la bande sahélienne située entre les latitudes $11^{0} 40$ et $16^{0} 31$ Nord et les longitudes $3^{0} 59$ et $13^{0} 40$ Est. Cette zone couvre $10 \%$ du territoire nigérien et reçoit en moyenne 300 à $700 \mathrm{~mm}$ de pluie par an. Le climat est de type tropical aride avec une saison de pluies de juin à septembre et une saison sèche d'octobre à mai (Figure 1). C'est une zone propice à l'agro-pastoralisme.

\section{Echantillonnage et collecte}

La population cible de cette étude était celle de la région de Maradi, Tahoua et Tillabéri réparties dans six (6) communes et dix-huit (18) villages. C'est un type d'enquête à échantillonnage stratifié à deux niveaux : les unités primaires (UP) sont les villages et les unités secondaires (US) les chefs d'exploitations agricoles familiales. La base de sondage pour le tirage des UP était constituée de la liste des villages dans la zone de l'étude. Un dénombrement a été effectué dans chaque village échantillonné en vue de constituer la base de sondage pour les US. La détermination de la taille de l'échantillon a pris en compte le taux de non réponses attendues et l'effet de grappe. Ainsi, la taille de l'échantillon des unités primaires est de 18 villages sur 90 villages soit $20 \%$. Ces villages ont été sélectionnés de façon raisonnée suivant les critères suivants: (i) accessibilité, (ii) proximité avec des marchés et existence d'un niveau minimum d'infrastructures, (iii) potentiel biophysique, (iv) potentiel organisationnel en termes de niveau d'organisation des paysans d'hommes, de femmes, de jeunes agriculteurs, d'éleveurs et d'agro éleveurs et (v) la récurrence aux crises alimentaire et nutritionnelle. Dans chaque village, une moyenne de 27 chefs d'exploitation par village a été enquêté suivant un tirage systématique simple dont l'algorithme est le suivant: (1) numérotation les chefs d'exploitation de 1 à $\mathrm{N}$; (2) calcule le pas de tirage $\mathrm{p}=\frac{\mathrm{N}}{\mathrm{n}}$; avec : $\mathrm{N}=$ nombre total des exploitations et $n=$ nombre d'exploitation choisi; (3) tirage aléatoire d'un nombre compris entre 1 et $p$ ( 1 et $p$ inclus). Soit $u_{1}$ ce nombre. C'est le numéro de la première exploitation tirée. Les autres exploitations sont retrouvées en ajoutant le pas $\mathrm{p}$ au dernier numéro tiré. Conduite suivant la méthodologie SMART, c'est une méthode d'enquête rapide, standardisée et simplifiée avec saisie en temps réel des données sur le terrain. Des Smartphones (tablettes) ont été utilisés pour la collecte des données sur le système digitalisé Open Data Kits « ODK v1.15.1 ».

Deux outils de collecte des données ont été utilisés: un guide d'entretien semistructuré et des questionnaires individuels. Au cours des entretiens semi-structurés organisés en focus groups, les informations collectées ont concerné les difficultés ou menaces qui reviennent fréquemment au niveau de leur communauté rurale, la classification des trois premières difficultés majeures pour chaque village, les relations entre la population et les autorités compétentes en cas de fléau (climatiques et socio-économiques) et les équipements et outils de travail collectifs. Quant aux enquêtes individuelles, elles ont consisté à recueillir les informations le lien entre les risques pluviométriques et l'insécurité alimentaire et leurs conséquences socioéconomiques sur chaque ménage enquêté.

Pour la priorisation des trois premières difficultés en lien avec l'insécurité alimentaire, nous avons, d'abord procédé à une Méthode Accélérée de Recherche Participative (MARP) en faisant l'arbre à problème de chaque village. Ensuite, pour le calcul des proportions, nous avons demandé à chaque chef d'exploitation agricole familiale de dire sa difficulté première, 
deuxième et difficulté troisième. Chaque chef d'exploitation a donné l'impact des difficultés sur la situation alimentaire et nutritionnel de son ménage.

\section{Analyses des données}

Les données ont été analysées au moyen des logiciels SPSS.20 et Munitab.18. Une analyse statistique descriptive et multivariée a été d'abord réalisée pour déterminer les principales caractéristiques pluviométriques de chaque région (Moyenne, les dispersions (écart-type), maxima, minima, les variations (coefficients de corrélation) et les fréquences et probabilités pour déterminer la significativité de la pluviométrie de chaque région. Cette analyse a permis de comprendre les caractéristiques des variabilités (risques) pluviométriques et la variable qui pèse sur la situation alimentaire et nutritionnelle des ménages ruraux. Ensuite, une analyse qualitative des données issues des entretiens semi-structurés pour répertorier et classer les menaces.
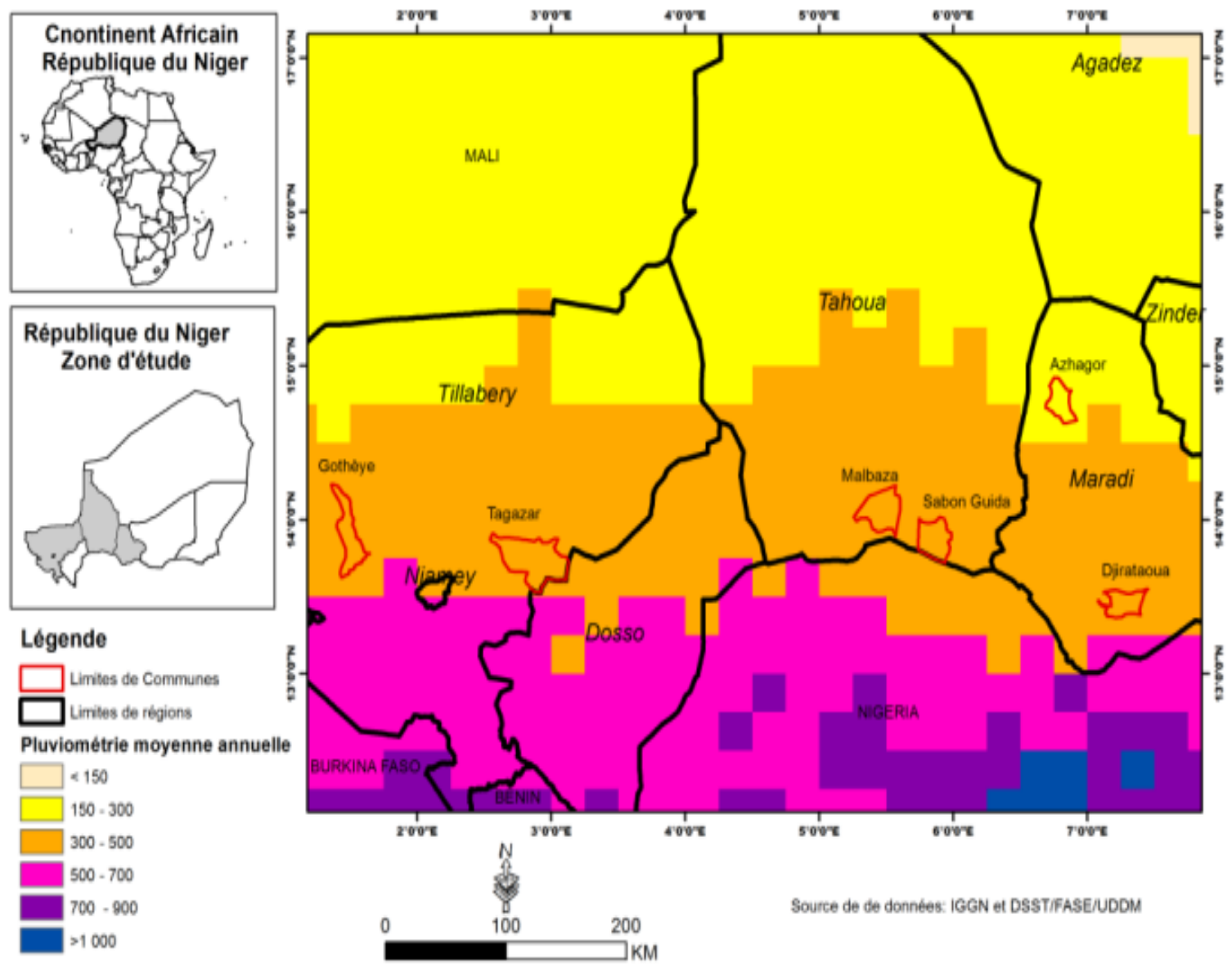

Figure 1 : Localisation de la zone d'étude. 


\section{RESULTATS}

\section{Analyse statistique de la pluviométrie}

D'une manière générale, la pluviométrie est significative $\mathrm{P}$-value est égal à $0,00(<5 \%)$. Mais les lettres différentes $(a, b$ et c) expliquent une différence significative entre la pluviométrie des trois régions. Les pluies des régions de Maradi et Tahoua sont significatives, P-value respectivement égale à 0,00 et 0,02 . Dans la région de Maradi, la pluviométrie varie entre $313,60 \mathrm{~mm}$ et 615,70 $\mathrm{mm}$ avec une moyennetécart-type de $502,32 \pm 81,3$ avec un coefficient de variation $16,18 \%$. Cette pluviométrie varie entre 218,32 à $689,90 \mathrm{~mm}$ avec une moyenne \pm ćcart-type de $429,89 \pm 110,98$ pour la région de Tillabéri avec un coefficient de variation de $25,81 \%$. Pour la région de Tahoua, la pluviométrie varie entre $204,74 \mathrm{~mm}$ et $634,40 \mathrm{~mm}$ avec une moyenne \pm écart-type de $362,84 \pm 88,9$ et un coefficient de variation $24,50 \%$ (Tableau 1).

\section{Analyse caractéristique de la répartition de la pluviométrie de chaque région de 1987 à 2017}

La pluviométrie des trente dernières années est repartie dans les trois régions selon les années, $1987 ; 1988 ; 1989 ; 199 ; 199$; $1992 ; 1995 ; 1996$; $1997 ; 1999$; 2000; 2001 ; 2017; $2004 ; 2006$; 2011 et 2009 pour la région de Maradi. Quant à la région de Tahoua, elle est caractérisée seulement par les pluviométries des $1991 ; 1994$ et 2002. Enfin la région de Tillabéri est marquée par les pluviométries des années : $1993 ; 1998$; 2003 ; $200 ; 2010 ; 2012 ; 2013 ; 2014 ; 2015 ; 2016$ et 2017 (Figure 2).

\section{Analyse des fréquences des risques pluviométriques}

Au cours de la période 1987 à 2017, l'évolution de la pluviométrie est marquée par des déficits et excédents pluviométriques par rapport aux moyennes de chaque région. $\mathrm{La}$ région de Maradi était de 15 sur 15 (15 années déficitaires et 15 années excédentaires) contre 17 sur 13 pour la région de Tahoua et 19 sur 11 pour Tillabéri. En moyenne 17 années sont déficitaires et 13 excédentaires au cours des 30 dernières années (Figure3).

Analyse des difficultés (menaces) des populations rurales au cours des dix dernières années

Les difficultés sont d'ordres climatiques, agronomiques et socioéconomiques énumérées par les populations rurales des trois régions (Tableau 2). Les difficultés climatiques sont, l'irrégularité des pluies, pluies tardives/pluie précoces et des arrêts précoces/tardifs, les inondations, les attaques des ravageurs et insectes sur les cultures pluviales, les vents, les poussières sont réciproques au niveau des trois régions. Quant aux difficultés agronomiques, ce sont, les attaques des oiseaux ravageurs et des insectes, les attaques des chenilles, les ressemis, les manques d'intrants et matériels agricoles. Enfin les difficultés socio-économiques diffèrent d'une région à l'autre mais l'augmentation des prix des céréales est la véritable difficulté des populations rurales des dix dernières années.

\section{Priorisation des trois premières difficultés majeures par les populations rurales}

Trois difficultés majeures ont été priorisés au niveau de chaque village (Tableau 3) pour faire le lien entre les difficultés et l'insécurité alimentaire et nutritionnelle. Les résultats ont montré que pour la $1^{1 \text { èr }}$ difficulté, la sécheresse occupe $(78,22 \%)$; la $2^{\text {ème }}$ difficulté, attaques et/maladies sur les cultures agricoles $(58,16 \%)$ et la $3^{\text {ème }}$ difficulté, augmentation des prix des céréales locaux $(88 \%)$.

\section{Analyse des causes de perte de production agricole céréalière}

Les résultats confirment que $71,1 \%$ sont causées par les faibles pluviométries et les inondations avec respectivement $46,3 \%$ pour les faibles pluviométries et $24,8 \%$ pour les inondations. Les attaques et/ou maladies $(24,3 \%)$ et le manque d'intrants et de matériels agricole $(3,93 \%)$ avec disparités régionales (Tableau 4). 
Analyse de l'impact des risques sur la sécurité alimentaire et nutritionnelle des ménages ruraux

Les résultats ont montré que les risques ont d'impacts différents sur la situation alimentaire et nutritionnelle des ménages ruraux. L'impact est très important avec un pourcentage moyen de 47,17 dont Maradi
$(56,4 \%)$, Tahoua $(48,2 \%)$ et Tillabéri $(36,9 \%)$. Impact modéré d'une moyenne de 35,47\% dont Maradi (30\%), Tahoua (48,3\%) et Tillabéri $(28,1 \%)$. Faible avec une moyenne de $17,36 \%$ dont Maradi (13,6\%), Tahoua (3,5\%) et Tillabéri (17,36\%). Pas d'impact zéro pourcent $0 \%$ pour toutes les régions (Tableau 5 ).

Tableau 1 : Analyse statistique de la pluviométrie des régions de Maradi, Tahoua et Tillabéri.

\begin{tabular}{llll}
\hline Pluviométries moyennes annuelles & Maradi & Tahoua & Tillabéri \\
\hline Moyenne \pm Ecart-type (mm) & $502,32 \pm 81,30 \mathbf{a}$ & $362,84 \pm 88,90 \mathbf{c}$ & $429,89 \pm 110,98 \mathbf{b}$ \\
\hline Coefficient de variation (\%) & 16,18 & 24,50 & 25,81 \\
\hline Minimum (mm) & 313,60 & 204,74 & 218,32 \\
\hline Maximum (mm) & 615,70 & 634,40 & 689,80 \\
\hline P-value & $0,00^{* * *}$ & $0,02 * * *$ & 0,09
\end{tabular}

Les valeurs suivies des lettres différentes sont statistiquement différentes au seuil de 5\% du test de Newman-Keuls. *** corrélation hautement significative.

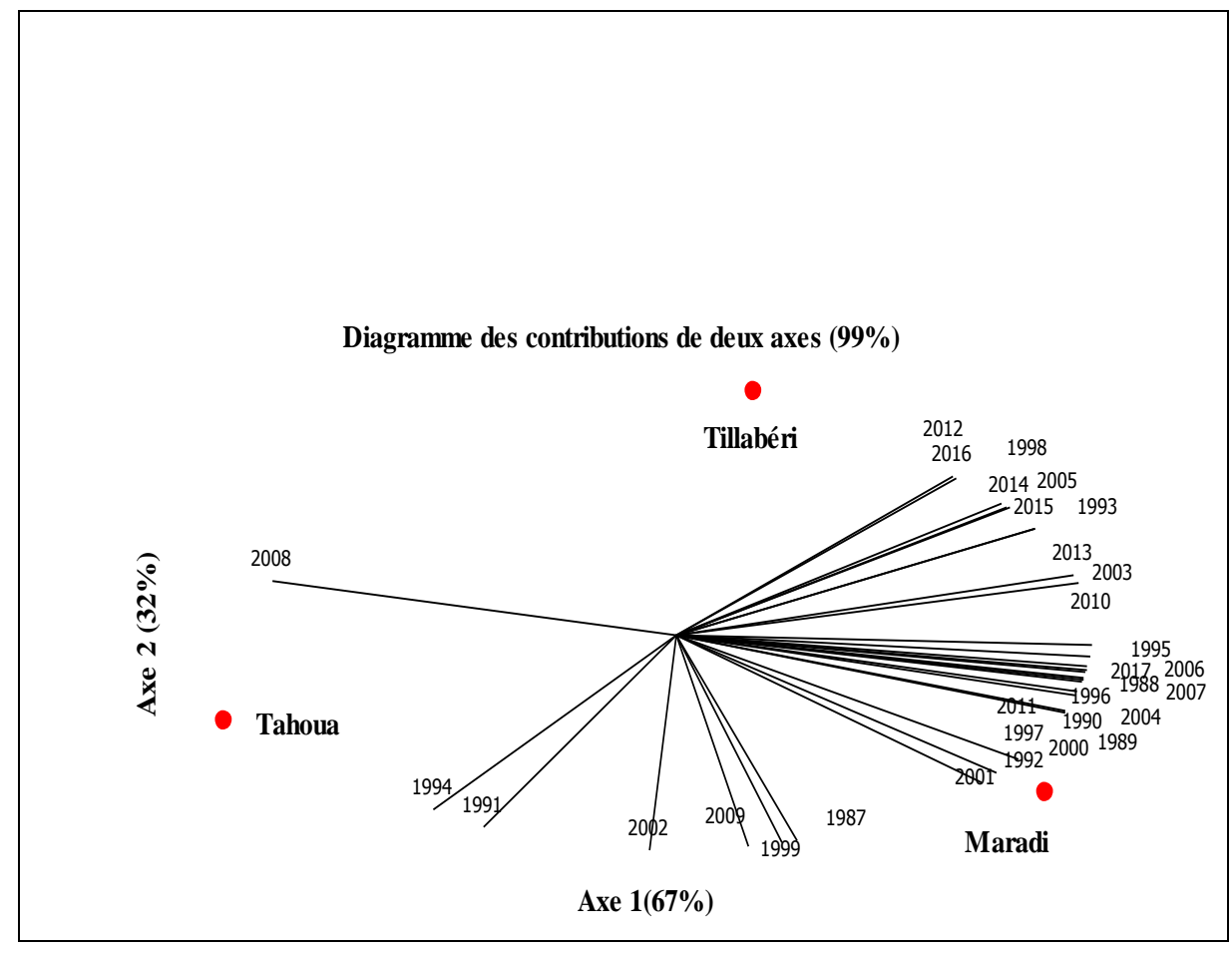

Figure 2: Répartition de la pluviométrie des trente dernières dans les trois régions de 1987 à 2017. 


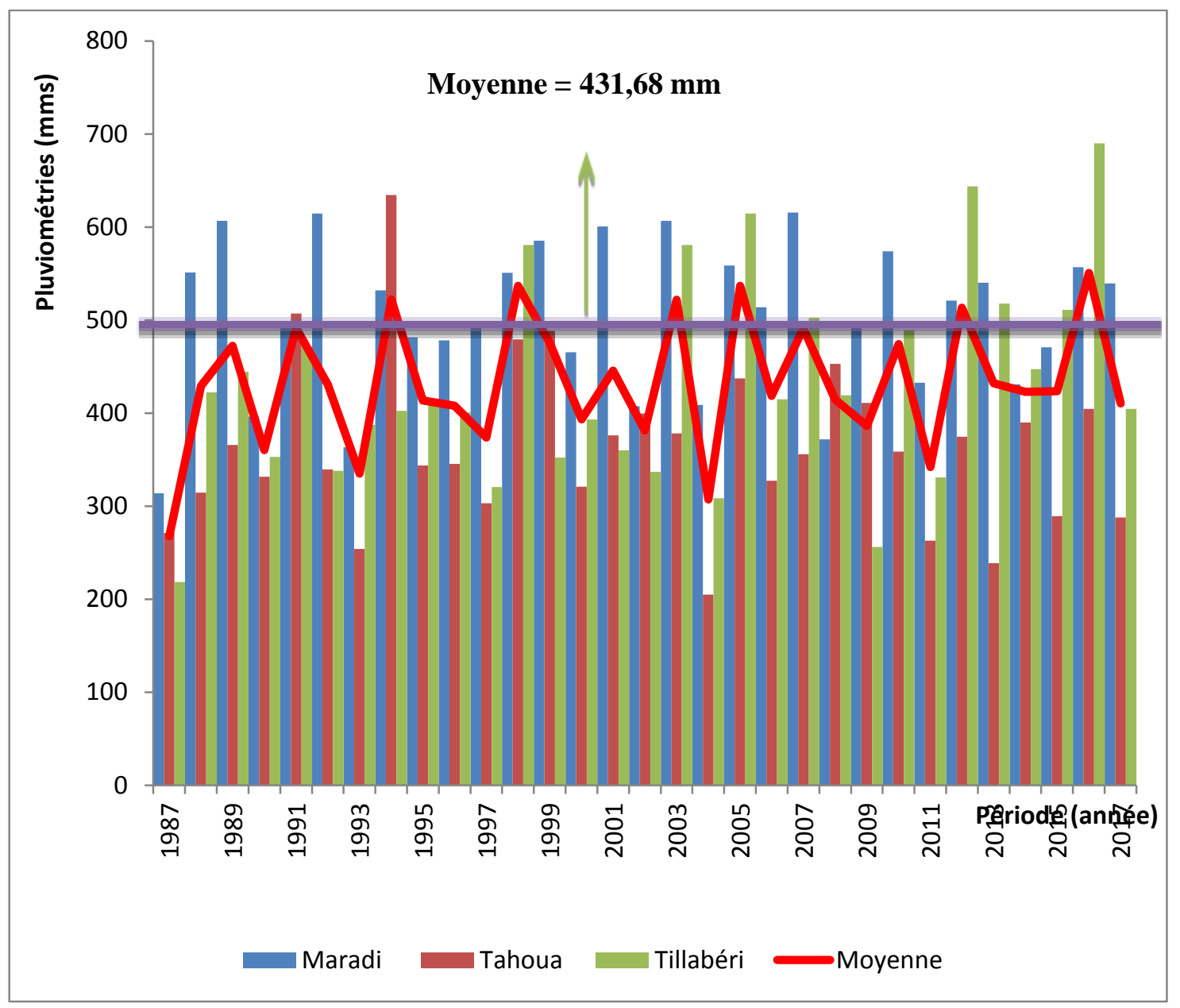

Figure 3: Evolution des cycles des extrêmes de la pluviométrie moyenne annuelle de 1987 à 2017.

Tableau 2 : Fréquence (\%) sur la situation alimentaire et nutritionnelle des ménages.

\begin{tabular}{llllc}
\hline $\begin{array}{l}\text { Fréquence (\%) impact des risques } \\
\text { sur la situation alimentaire et } \\
\text { nutritionnelle }\end{array}$ & $\begin{array}{l}\text { Très } \\
\text { important }\end{array}$ & Modéré & Faible & Pas d'impact \\
\hline Maradi & 56,4 & 30 & 13,6 & 0 \\
\hline Tahoua & 48,2 & 48,3 & 3,5 & 0 \\
\hline Tillabéri & 36,9 & 28,1 & 35 & 0 \\
\hline Moyenne & 47,17 & 35,47 & 17,36 & 0 \\
\hline
\end{tabular}


Tableau 2 : Difficultés des chefs d'exploitations agricoles durant les dix dernières années.

\begin{tabular}{|c|c|c|}
\hline $\begin{array}{l}\text { Difficultés au } \\
\text { cours des } 10 \\
\text { dernières } \\
\text { années }\end{array}$ & Maradi & Tahoua \\
\hline
\end{tabular}

$\begin{array}{lll} & \begin{array}{l}\text { Irrégularité des pluies, baisse } \\ \text { des pluies, }\end{array} & \begin{array}{l}\text { Irrégularité des pluies, } \\ \text { baisse des pluies, arrêt et } \\ \text { arrêt et tardif des pluies, } \\ \text { pluies précoces, pluies } \\ \text { tardives, }\end{array} \\ \text { tardif des pluies, pluies } \\ \text { précoces, pluies tardives, } \\ \text { vents, poussière, canicule, } \\ \text { inondation. }\end{array}$

Irrégularité des pluies, baisse des pluies, arrêt et tardif des pluies, pluies précoces, pluies tardives, vents, poussière, canicule, inondation, baisse du niveau d'eau du fleuve, ensablement et évaporation accélérée des eaux du fleuve.

Baisse des récoltes
Attaques des ravageurs
(chenilles sur le mil et le
niébé),

Agronomiques- Érosion éolienne et hydrique. écologiques Ressemis, perte de semence Plusieurs maladies des animaux, sous-produits animaux non rentables.

Augmentation des prix des
denrées alimentaires,
augmentation des céréales
locaux, augmentation des prix
des intrants

Socioéconomiques
Baisse des prix des animaux, mévente, conflits agriculteurs éleveurs.

\section{Manque d'intrants et de matériels agricoles (manque d'engrais de qualité) ; attaques acridiennes et de ravageur, Sols argileux, baisse des récoltes. Diminution du maraichage.}

Attaques des ravageurs (criquets, oiseaux), chenille sur le niébé et le gombo, Ravageurs aquatiques sur les cultures maraichères, sur les manguiers et les clôtures des jardins. Ressemis, perte de semence.
Augmentation des prix des denrées alimentaires, augmentation de céréales locales augmentation du prix du carburant et du transport, Augmentation des semences d'oignon et d'engrais
Augmentation des prix des denrées alimentaires, augmentation du prix du mil, augmentation du prix du transport, baisse des revenus, conflits agriculteurs éleveurs, conflits Homme-Faune (attaque des hippopotames).
Conflit de génération (jeunes- vieux).

Tableau 3: Fréquence (\%) des trois premières difficultés source d'insécurité alimentaire et nutritionnelle.

\begin{tabular}{lccc}
\hline Trois 1ères difficultés & Sécheresse & Fréquence (\%) & \\
\hline Attaques et/ou maladies & $\begin{array}{c}\text { Augmentation des prix des } \\
\text { céréales locaux }\end{array}$ \\
\hline $1^{\text {ère }}$ difficulté & 78,22 & 21,78 & 0 \\
\hline $2^{\text {ème }}$ difficulté & 20,44 & 58,16 & 12 \\
\hline $3^{\text {ème }}$ difficulté & 1,34 & 20,06 & 88 \\
\hline Ensemble & 100 & 100 & 100 \\
\hline
\end{tabular}


Tableau 4 : Fréquence (\%) des causes de diminution de la production agricole des céréales.

\begin{tabular}{lcccc}
\hline $\begin{array}{l}\text { Causes de diminution de la } \\
\text { production agricole céréalière }\end{array}$ & Maradi & Tahoua & Tillabéri & Ensemble \\
\hline Faible Pluviométrie & 47,1 & 42,6 & 34,9 & 40,4 \\
\hline Insectes et/ou maladies & 25,7 & 34,4 & 12,8 & 22,1 \\
\hline Manque d'intrants agricoles & 2,9 & 1,6 & 7,3 & 4,6 \\
\hline Arrêt précoces/tardif des pluies & 5,7 & 4,9 & 3,7 & 4,6 \\
\hline Inondations & 0,0 & 0,0 & 1,8 & 0,8 \\
\hline Autres & 18,6 & 16,4 & 39,4 & 27,5 \\
\hline Total & 100 & 100 & 100 & 100 \\
\hline
\end{tabular}

Tableau 5 : Fréquence d'impact de deux risques sur la situation alimentaire et nutritionnelle des ménages exploitations agricoles.

\begin{tabular}{lllll}
\hline $\begin{array}{l}\text { Fréquence (\%) impact des risques sur } \\
\text { la situation alimentaire et } \\
\text { nutritionnelle des ménages }\end{array}$ & $\begin{array}{l}\text { Très } \\
\text { important }\end{array}$ & Modéré & Faible & Pas d'impact \\
\hline Maradi & 56,4 & 30 & 13,6 & 0 \\
\hline Tahoua & 48,2 & 48,3 & 3,5 & 0 \\
\hline Tillabéri & 36,9 & 28,1 & 35 & 0 \\
\hline Moyenne & 47,17 & 35,47 & 17,36 & 0 \\
\hline
\end{tabular}

\section{DISCUSSION}

Les résultats montrent, d'une part, des variabilités pluviométriques interannuelles mal réparties se caractérisant beaucoup plus par des déficits. Ces derniers de plus en plus fréquents sont pour la plupart la cause des baisses de la production agricole céréalière et l'augmentation des prix des céréales locales. Ces résultats sont conformes à ceux de Habou et al. (2016) qui ont montré que la performance du secteur agricole est néanmoins très instable du fait de sa forte exposition aux changements climatiques. Ainsi, les résultats de (Cabral, 2102) ont montré que la tendance de la pluviométrie en Afrique est à la baisse. L'étude, a démontré que, la baisse de la consommation céréalière par tête a été particulièrement grave durant les périodes de grandes sécheresses. Cette baisse de pluviométrie ne peut qu'avoir des conséquences sévères sur la croissance économique. Pour le même auteur, un choc, tel qu'une sécheresse a des effets importants sur la production agricole et la demande des facteurs. Il affecte également les prix à la consommation, au-delà un choc pluviométrique aura des implications sur la pauvreté. Les sécheresses ont des effets rétroactifs et affectent dangereusement toute l'économie.

Partant de la même idée, Jouve et al. (2012), confirment que les sécheresses font flamber les prix des céréales directement en campagne et indirectement en ville par les produits transformés comme le pain, la farine, la semoule). La principale raison de la flambée des prix des céréales est liée à la météorologie et la sécheresse qui font accroitre le cours des céréales. Certains auteurs sont allés au-delà, en comparant divers écotypes. Les résultats ont 
relevé que la variabilité intra climatique est plus élevée chez les céréales et les cultures potagères pluviales tandis qu'elle est moins élevée chez les cultures maraichères (Bambara, 2011).

Ces résultats sont différents d'abord de ceux Jenny (2010), qui montre qu'au Niger, la souffrance causée par une période de mauvaise récolte a été associée à une flambée dramatique des prix alimentaires profitant à une communauté de commerçant en plein essor qui n'ont pas connu de contrôle étatique des prix dans les années récentes. Ensuite, Kaminski et al. (2013) ont montré que les prix du mil et du sorgho varient d'une région à l'autre et sont basses au niveau des zones de productions importantes. Ainsi, l'étude a démontré que les marges sont basses dans les zones de grosse production en raison de la concurrence et des économies d'échelle et beaucoup plus élevées dans des parties enclavées enregistrant une production modeste. Enfin, selon ces auteurs la filière sorgho-mil a des segments de transformation et de conditionnement insuffisamment développés alors que les marchés connaissent une fluctuation des prix d'une saison à l'autre, imputable à des fortes variations de l'offre et de la qualité et à la capacité limitée de stockage. Les prix des céréales sèches notamment le mil et le sorgho ont subi une variabilité interannuelle élevée, sans aucune tendance significativement détectable. Il existe une relation linéaire entre les deux séries de prix, le prix aux producteurs justifiant une part à peu près constante du prix aux consommateurs dans les villes. En effet, les risques pluviométriques fait augmenter l'importation du riz car la production agricole étant faible et ne couvre que $47 \%$ des besoins des populations (Traoré, 2015).

Les résultats ont montré que les attaques et/ maladies engendrant des pertes de production agricole corroborent avec les sécheresses, ces résultats sont similaires à une étude du centre Agrhymet montrant que les déficits de production du mil et du sorgho ont été observés durant les années à faibles précipitations. Les quantités obtenues sont en dessous des besoins de consommation minimum (Véronique, 2014). Confirmant la même idée (Jenny, 2010) a montré que les sécheresses au Niger sont fortement corrélées à des mauvaises récoltes, une hausse de prix des produits vivriers et une baisse des revenus des populations. Par ailleurs, il démontre également que les sécheresses sont fortement corrélées aux crises alimentaires et aux famines. Dans une étude de synthèse bibliographique, Kadri et al. (2019) montrent que, malgré son importance, la croissance et la productivité du mil sont entravées par l'incidence des bioagresseurs (maladies et organismes nuisibles). Parmi, les maladies, celles qui causent plus des dégâts dans la production du mil sont les maladies fongiques.

Les résultats de la présente étude montrent d'autre part que les producteurs ont affirmé que les pertes de production dues aux attaques acridiennes et des ravageurs sont plus lourdes que celles des chenilles et/ou maladies. Les pertes globales dues à ces ravageurs et maladies sont considérables et peuvent être quantitatives ou qualitatives (Sall, 2015). Les pertes de production dues aux attaques acridiennes sont estimées à $1 / 3$ de la récolte et 10 à $30 \%$ pour les oiseaux ravageurs sur les variétés précoces qui mûrissent vite (Habou, 2016 ; Kadri, 2019). Confirmant la même idée ces auteurs ont relevé que l'invasion acridienne de 2005 a soldé le bilan céréalier du Niger à un déficit céréalier négatif de 278.350 tonnes suite à l'affectation sévère de la production agricole dans les régions du Nord et sud par les attaques acridiennes. Les pertes de la production céréalière et fourragère étaient estimées à $26 \%$ (FAO/PAM, 2004). Conformément aux dires des producteurs la sécheresse est un phénomène dévastateur qui crée des chocs. Elle entraine, contrairement aux catastrophes à occurrence rapide, la ruine lente de la région et installe son œuvre de destruction avec le temps. Le phénomène dure des années et peut avoir des ravages sur l'agriculture et l'approvisionnement en eau (François, 2012). Bidou et al. (2006) confirment que les difficultés alimentaires plus sévères sont liées à des sécheresses périodiques et à des invasions acridiennes.

Au demeurant, pour faire le suivi de ces risques pluviométriques en lien l'insécurité 
alimentaire et nutritionnelle, plusieurs outils dont la courbe de Maxwell permet de faire la combinaison des stratégies observées au moins deux fois au cours des sept derniers jours par les ménages en quatre types qu'il convient de croiser avec les quatre classes d'insécurité alimentaire. Il y'a aussi le «Cadre Harmonisé », outil de dernière génération inventé le Comité Inter-permanent de Lutte contre la Sécheresse au Sahel (CILSS), qui est un d'outil d'analyse et d'indentification des risques.

Cette étude, a également montré que l'alternance des risques pluviométriques consécutifs durant les trente dernières années, fait que les populations oscillent entre sécheresses et/ou inondations.

Nonobstant, les inondations qui ont été citées parmi les causes de baisse de production, ces inondations sont également parmi les risques pluviométriques qui préoccupaient les producteurs ruraux. Cela s'explique en partie par les quantités de pluies enregistrées ces dernières années $(2003 ; 2006 ; 2012$ et 2016) qui dépassent largement les moyennes créant des ruissellements importants dépassant le niveau du fleuve, d'où les inondations. Pour Daouda et al. (2018) ; les inondations sont de plus de plus fréquentes et dramatiques. Elles sont certes liées aux fortes pluies enregistrées, cependant, les dernières inondations sont surtout amplifiées par l'effet des pluies consécutives et qui sont plus agressives. Vieri et Maurizio (2016), dans une évaluation multirisque, prévoirait des risques d'inondation à partir des horizons 2025 et 2050 qui aggraveront plus la vulnérabilité alimentaire et nutritionnelle. Cette dernière sera accentuée par l'urbanisation qui a permis l'occupation des zones de ruissellements. Ces résultats ont été appuyés par le PRESAO qui a déjà prévu dans la zone sahélienne des pluies normales à tendance humide au Sahel et Afrique de l'ouest. Ces inondations n'épargnent pas les moyens d'existence des populations ce qui fragilise encore leur état de vulnérabilité alimentaire. Ouedrago et al. (2011) ont conclu qu'en Afrique sub-saharienne les calamités naturelles telles que: les sécheresses, les inondations, les criquets, guerres occasionnent de perturbations considérables sur le niveau de production. Ces calamités constituent «un facteur de production » dans l'agriculteur. $\mathrm{Ce}$ facteur prend la valeur 1 s'il y'a des calamités qui affectent négativement la production. Elles ont pour conséquences les perturbations alimentaires.

Contrairement à Jodelle (2007) et Gado (2010), les crises alimentaires constituent des événements dont la dimension sociale est fréquemment négligée « on oublie trop souvent que les famines ne doivent pas être essentiellement considérées comme des problèmes purement techniques ou comme une conséquence inévitable des accidents climatiques. L'auteur détermine les différents facteurs sociaux entrés en jeu dans les crises et expose la manière dont celles-ci ont été vécues jusqu'ici au niveau de la paysannerie. A cela s'ajoute, le disfonctionnement des dispositifs nationaux de prévention et prise en charge des urgences des crises alimentaires et les conflits armés qui aggravent l'insécurité alimentaire et Nutritionnelle (Baoua, 2014 ; Boubé, 2017).

\section{Conclusion}

La présente étude a permis de confirmer l'assertion selon laquelle la connaissance des risques pluviométriques et l'insécurité alimentaire tient une place importante dans le savoir paysan pour s'adapter aux contraintes de leur milieu de vie. Elle a montré que les paysans perçoivent clairement les risques qui essayent leurs systèmes de production. Les perceptions paysannes des risques corroborent en effet, la littérature sur les risques climatiques et socio-économiques dans le contexte du Sahel. La recrudescence des poches de sécheresse et la fluctuation des prix des céréales relevées par les paysans constituent des phénomènes réels de la crise alimentaire et nutritionnelle et de la vulnérabilité de manière globale au Sahel. Par contre à l'intérieur d'un même village, les réponses apportées face aux effets négatifs de la sécheresse et de la fluctuation des prix des céréales ainsi que les modalités de leur mise en œuvre diffèrent selon la capacité d'investissement du ménage et de sa catégorie 
socio-économique. La production agricole et durable dépendra non seulement de la pluviométrie mais également des conditions agro-écologiques stables et de la technicité des producteurs en passant par une utilisation des variétés de semences résilientes aux risques climatiques et une amélioration de la fertilité des sols. L'économie agricole est tributaire à la stabilité des variabilités pluviométriques mais aussi agro-écologiques. La pluviométrie a une forte influence dans le déterminisme de l'insécurité alimentaire. La conversion des exploitations agricoles familiales traditionnelles en exploitations familiales intégrées résilientes aux variabilités pluviométriques est une nécessité impérieuse. Un regard doit être aussi porté sur les menaces des hippopotames, les conflits agriculteurs éleveurs et les attaques des bandits qui sont parfois transversaux aux crises alimentaires et nutritionnelles.

\section{CONFLITS D'INTERETS}

Les auteurs de ce manuscrit déclarent qu'ils n'ont pas de conflits d'intérêt entre eux.

\section{CONTRIBUTIONS DES AUTEURS}

Dans la réalisation de la présente étude, LS, AMM et AMZ ont contribué à l'élaboration des outils de collecte des données et à la relecture du document. IS a participé à la relecture du document. MM a apporté des conseils sur les traitements des données. En outre LS et AMS ont apporté des conseils dans la collecte et le traitement des données. Ils ont tous approuvé la version finale du document.

\section{REMERCIEMENTS}

Les auteurs remercient le projet de Recherche-Développement pour la Sécurité Alimentaire et l'adaptation au changement des systèmes ruraux de production (REDSAACC), l'Institut National de la Recherche Agronomique (INRAN) pour le partenariat grâce auquel ce travail a été financé. Les universités Boubakar Bâ de Tillabéri (UBBT) et l'Université Dan Dicko Dankoulodo de Maradi (UDDM) pour leur partenariat technique et financier.

\section{REFERENCES}

Afrique Verte AMMA. 2013. Quelques maladies et ravageurs du mil, sorgho et riz. Fiche de formation, 6.p.

Bacci M, Katiellou LG, Diolla LA. 2014. Prévention des catastrophes et développement agricole pour la sécurité alimentaire. Rapport d'étude ANADIA Niger, 26.p.

Bahari IM, Ibrahim B M, et Oumarou F M. 2018. Évolution des caractéristiques pluviométriques et recrudescence des inondations dans les localités riveraines du fleuve Niger. VertigO, 18(1). URL: http://journals.openedition.org/vertigo/19 891.

Bambara D, Bilgo A, Lompo F, Hien V. 2011. Influence du changement climatique sur la diversité inter et intra-spécifique des plantes cultivées à Tougou au Nord du Burkina Faso. Int. J. Biol. Chem. Sci., 5(6): 2415-2433. DOI: http://dx.doi.org/10.4314/ijbcs.v5i6.21

Banque Mondiale. 2011. Profil et déterminant de la pauvreté au Niger. Rapport INS, p. 37.

Baou I, Ali M, Balla A, Adam T. 2014. Systèmes locaux d'alerte précoce et de réponse aux urgences: motivation et partenariat exigeant. Journal of Applied Biosciences, 85(1): 7740-7750. DOI: http://dx.doi.org/10.4314/jab.v85i1.2

Bidou JE, Droy I, Rasolofo P. 2006. Risque climatique et vulnérabilité alimentaire dans le sud Madagascar: question d'échelle, questions de méthodes. Rapport dc consultation, 6.p.

Boubé N. 2017. Les djihadistes au Sahel: enjeux et perspectives. Afrique et Développement, 42(3):197-217. DOI : https://www.jstor.org/stable/90018141

Boussard JM. 2010. Pourquoi l'instabilité estelle une caractéristique structurelle des marchés agricoles? Economie rurale|CAIRN.INFO, 2010/6 (320): 6983. DOI : https://www.cairn.info/revueeconomie-rurale-2010-6-page-69.htm\#.

Douada A, Tachiana V, Tiepolo M. 2016. Milieu physique, peuplement et 
exposition aux aléas hydro-climatiques dans la région de Tillabéri, Niger. ResearchGate, 2007a (21): 27-50. DOI: https://www.researchgate.net/publication /316789257.

FAO. 2020. FAO en situation d'urgences: de la prevention à une meilleure reconstruction.

http://www.fao.org/emergencies

FAO/PAM. 2004. Mission d'évaluation des récoltes et des disponibilités alimentaires au Niger. Rapport spécial.6.p.

François JC. 2012. Impact des aléas pluviométriques sur les disparités régionales de pauvreté au Sénégal. Revue Economie de développement CAIRN.INFO., 1(20): 69-95. DOI : https://doi.org/10.3917/edd.261.0069.

Gado BA. 2010. Crises Alimentaires en Afrique Sahélienne: Les Réponses Paysannes. Edit. Flamboyant, p.210.

Habou ZA, Boubacar KM, Adam T. 2016. Les systèmes de productions agricoles du Niger face aux changements climatiques : Défis et perspectives. Int. $J$. Biol. Chem. Sci., 10(3): 1262-1272. DOI: http://doi.org/10.4314/ijbcs.v10i3.28.

Haougui A, Basso A, Demas P. 2017. La cicadelle écumeuse des céréales. Fiche technique. INRAN/RECA/UAM.p.2.

Idrissa F. 2008. Impact des fluctuations pluviométriques sur la production dans la région de Thionck-Essyl en bassa Casamance. Mémoire de fin d'étude pour l'obtention d'un certificat d'aptitude à l'enseignement moyen. p.42.

Jenny CA. 2010. Chocs pluviométriques, marchés et crises alimentaires : l'effet de la sécheresse sur les marchés céréaliers du Niger. Revue d'Economie de Développement|CAIRN.INFO., $\mathbf{1}(18)$ : 71-108.

DOI : https://doi.org/10.3917/edd.241.0071.

Jodelle TD. 2007. Les débats autour de la crise alimentaire du Niger en2005: Quels enjeux relèvent-ils pour les acteurs humanitaires au Niger? Mémoire de
Master (CIAHPD). Université Paris I, Sorbonne.p.87.

Jouve AM, Kheffache Y, Belgazi S. 2012. La filière des céréales dans les pays du Maghreb : constante des enjeux, évolution des politiques. CIHEAM Série B (14) :169-192. DOI : https://om.ciheam.org.php.article?DPDF $=$ CI960049.

Kadri A, Halilou H, Karimou I, 2019. Culture du mil [Pennisetum glaucum (L) R. Br] et ses contraintes à la production: une revue. Faso. Int. J. Biol. Chem. Sci., 13(1): 503524.

DOI: http://dx.doi.org/10.4314/ijbcs.v13i1.40

Kaminski J, Elbehri A, Samaké M. 2013. Une évaluation des filières du Sorgho et de mil les implications pour une politique cohérente de développement, Rapport de consultation. FAO/FIDA. p.525-547.

Kako N. 2000. L'Insécurité Alimentaire en Afrique Subsaharienne: le Rôle des Incertitudes. Edit. L'Harmattan; p.200212.

Ouédrago D, Kaboré M, Kienou B. 2007. Insécurité alimentaire, vulnérabilité et pauvreté en milieu rural au Burkina : une approche en termes de consommation en d'énergie. Mondes en Développement. 2007/4 (140): 65-84. DOI : 10.3917/med.140.0065.

Sall M. 2017. Les exploitations agricoles familiales face aux risques agricoles et climatiques: stratégies développées et assurance agricole. Thèse de Doctorat. Université de Toulouse. Toulouse, p.276.

Traoré A, Traoré K, Traoré O, Bado BV, Nacro BH, Sedogo MP. 2015. Int. J. Biol. Chem. Sci., 9(6): 2685-2697. DOI: http://dx.doi.org/10.4314/ijbcs.v9i6.14

Véronique C. 2014. Sahel: le régime des précipitations s'est intensifié au cours des 20 dernières années. Rapport d'étude AMMA-CATCH.2p.

Vieri T, Maurizio T. 2016. Risque et Adaptation Climatique dans la Région de Tillabéri au Niger. Edit. L'Harmatttan ; p.234-276. 